\title{
High levels of oxidation-reduction potential in frozen-thawed human semen are significantly correlated with poor post-thaw sperm quality
}

\author{
Ramadan Saleh $^{1,2}$ (D) | Mohamed Elsuity ${ }^{1}$ | Ralf Henkel ${ }^{3,4}$ (D) | Ashok Agarwal ${ }^{4}$ (D)
}

\author{
${ }^{1}$ Department of Dermatology, Venereology \\ and Andrology, Faculty of Medicine, Sohag \\ University, Sohag, Egypt \\ ${ }^{2}$ Ajyal IVF Center, Ajyal Hospital, Sohag, \\ Egypt \\ ${ }^{3}$ Department of Medical Bioscience, \\ University of the Western Cape, Bellville, \\ South Africa \\ ${ }^{4}$ Department of Urology, American Center \\ for Reproductive Medicine, Cleveland Clinic, \\ Cleveland, $\mathrm{OH}$, USA

\section{Correspondence} \\ Ramadan Saleh, Department of \\ Dermatology, Venereology and Andrology, \\ Faculty of Medicine, Sohag University, \\ Sohag 82524, Egypt. \\ Emails: salehr2010@yahoo.com; ramadan_ \\ saleh@med.sohag.edu.eg
}

\begin{abstract}
This study examined the relationship between oxidation-reduction potential (ORP) in frozen-thawed semen and the post-thaw sperm parameters. Levels of ORP were measured in 25 samples from men presenting for routine infertility work-up and were expressed as millivolt $(\mathrm{mV}) / 10^{6} \mathrm{sperm} / \mathrm{ml}$. Frozen-thawed samples were examined for post-thaw total motility (TM\%), progressive motility (PM\%), total sperm count (TSC) and ORP. The cryo-survival rate (CSR) was calculated as post-thaw TM/pre-freeze $\mathrm{TM} \times 100$. Data are provided as median and interquartile range $(25$ th and 75 th percentiles). The post-thaw TM\% (10.0\% [4.00\%, 15.1\%]), PM\% (5.88\% [2.97\%, 9.33\%]) and TSC $\left(12.5[10.0,17.5] \times 10^{6}\right.$ sperm) were significantly lower than the pre-freeze TM\% (45.9\% [32.9\%, 59.1\%], PM\% (31.5\% [24.4\%, 40.0\%] and TSC (120 [90, 250] $\times 10^{6}$ sperm $)(p<.001)$. Post-thaw ORP $\left(2.62[2.52,3.13] \mathrm{mV} / 10^{6} \mathrm{sperm} / \mathrm{ml}\right)$ was significantly higher than pre-freeze ORP $\left(0.73[0.54,1.21] \mathrm{mV} / 10^{6} \mathrm{sperm} / \mathrm{ml} ; p<.001\right)$. The CSR was $21.7 \%$ (11.3\%, 31.9\%). The post-thaw seminal ORP was negatively correlated with post-thaw TM\% ( $r=-.5 ; p=.02)$, PM\% ( $r=-.41 ; p=.03)$, TSC $(r=-.60$; $p=.03)$ and CSR $(r=-.52 ; p=.01)$. Increased levels of ORP are significantly correlated with poor post-thaw sperm quality and CSR.
\end{abstract}

\section{KEYWORDS}

cryo-survival rate, human semen, oxidation-reduction potential, sperm cryopreservation

\section{1 | INTRODUCTION}

Sperm cryopreservation has revolutionised the field of assisted reproduction, particularly when the infertile men are presenting with azoospermia (Anger, Gilbert, \& Goldstein, 2003). It allows those azoospermic men to avoid the need for repeated sperm retrieval surgeries. Sperm cryopreservation is also essential for fertility preservation in cancer patients who undergo chemotherapy, radiotherapy or radical pelvic surgery (Sharma, Kattoor, Ghulmiyyah, \& Agarwal, 2015).

Despite recent advances in the tools and techniques of cryopreservation, the quality of post-thaw spermatozoa is still suboptimal due to the inevitable cell injury of the freezing process (Medeiros, Forell, Oliveira, \& Rodrigues, 2002). Several factors had been proposed to explain the cryo-damage to human spermatozoa such as cold shock, osmotic stress and oxidative stress (OS; Amidi, Pazhohan, Shabani, Khodarahmian, \& Nekoonam, 2016). The post-thaw sperm quality is critically affected by the OS status that accompanies the cryopreservation process (Bucak et al., 2010; Kumar et al., 2018).

Freezing and thawing result in the generation of reactive oxygen species (ROS) in semen samples due to cold shock and dilution of seminal plasma (Watson, 2000). The susceptibility of spermatozoa to ROS-induced damage is due to the large content of polyunsaturated fatty acids (PUFA) in their plasma membranes (Alvarez \& Storey, 1995) and the small amount of scavenging antioxidants in their cytoplasm (Sharma \& Agarwal, 1996). Sperm damage due to excess ROS is mediated via reduction of motility and fertilising potential; 
and increased nuclear DNA fragmentation (Aitken, Buckingham, \& Harkiss, 1993).

Oxidation-reduction potential (ORP) has recently been introduced as a simple, accurate and reliable tool for quantitative estimation of OS levels in semen (Agarwal, Roychoudhury, Bjugstad, \& Cho, 2016). In this study, levels of ORP were assessed in the cryopreserved human semen, and their relationship to post-thaw sperm quality and cryo-survival rate (CSR) were determined.

\section{2 | MATERIALS AND METHODS}

The study was approved by the Research and Ethical Committees at Faculty of Medicine, Sohag University, Sohag, Egypt. The study was conducted in Ajyal IVF Center, Ajyal Hospital, Sohag Governorate, Egypt, between November 2016 and April 2017. Fresh semen samples were obtained from 25 men who attended the infertility clinic for initial fertility evaluation. Standard semen parameters that include sperm concentration $\left(\times 10^{6} / \mathrm{ml}\right)$, total sperm count $\left(\right.$ TSC $\left.\times 10^{6}\right)$, percentage of total motility (TM\%), percentage of progressive motility (PM\%), normal forms (\%) and seminal leucocyte counts $\left(\times 10^{6} / \mathrm{ml}\right)$ were measured in the neat (fresh) semen samples according to guidelines of the fifth Edition of the World Health Organization Manual (WHO, 2010). Samples with the diagnosis of azoospermia, severe oligozoospermia (sperm concentration $<1 \times 10^{6} / \mathrm{ml}$ ) or leucocytospermia (seminal leucocyte count $>1 \times 10^{6} / \mathrm{ml}$ ) were excluded.

\section{1 | Measurement of seminal ORP}

Levels of ORP were measured in the fresh semen samples and after cryopreservation using the MiOXSYS ${ }^{\circledR}$ System (Aytu BioScience) as previously described (Agarwal, Roychoudhury, et al., 2016). The levels of ORP were expressed as $\mathrm{mV} / 10^{6} \mathrm{sperm} / \mathrm{ml}$, and the cut-off value for normal seminal ORP was considered at the level $\leq 1.34 \mathrm{mV} / 10^{6}$ sperm/ml (Agarwal, Roychoudhury, et al., 2016; Agarwal, Sharma, Roychoudhury, Plessis, \& Sabanegh, 2016). A seminal ORP level higher than $1.34 \mathrm{mV} / 10^{6} \mathrm{sperm} / \mathrm{ml}$ indicates a state of OS.

\section{2 | Sperm cryopreservation}

Equal amounts of $0.5 \mathrm{ml}$ of fresh liquefied semen samples were frozen using the slow freezing technique (Sieme, Oldenhof, \& Wolkers, 2016). Sperm freezing medium (Life Global Group) was added to a fresh semen aliquot to reach a final dilution of 1:1. Thawing of frozen semen samples was done at $37^{\circ} \mathrm{C}$ after a fixed interval of 1 week following cryopreservation. Then, thawed semen samples were examined for the post-thaw TM\%, PM\%, TSC and seminal ORP levels. The CSR was calculated as post-thaw TM/ pre-freeze TM $\times 100$.
TABLE 1 Comparisons of sperm parameters and seminal ORP levels between pre-freeze and post-thaw semen samples $(n=25)$

\begin{tabular}{|llll|}
\hline Variable & Pre-freeze values & Post-thaw values & $p$ value \\
\hline $\begin{array}{c}\text { Total motility } \\
\text { (\%) }\end{array}$ & $45.9(32.9,59.1)$ & $10.0(4.00,15.1)$ & $<.001$ \\
\hline $\begin{array}{c}\text { Progressive } \\
\text { motility (\%) }\end{array}$ & $31.5(24.4,40.0)$ & $5.88(2.97,9.33)$ & $<.001$ \\
\hline $\begin{array}{c}\text { Normal } \\
\text { sperm } \\
\text { forms }(\%)\end{array}$ & $5.00(3.00,5.00)$ & $5.00(3.00,5.00)$ & .992 \\
\hline $\begin{array}{c}\text { Seminal ORP } \\
\text { (mV/10 } \\
\text { sperm/ml) }\end{array}$ & $0.73(0.54,1.21)$ & $2.62(2.52,3.13)$ & $<.001$ \\
\hline
\end{tabular}

Note: Data are presented as median and interquartile range (25th and 75th percentiles). A $p$ value $<.05$ was considered statistically significant. Abbreviation: ORP, oxidation-reduction potential.

\section{3 | Statistical analysis}

The data were analysed using SPSS version 24.0 (IBM Corp.). Normality of data distribution was checked by Shapiro-Wilk test. The data are presented as median and interquartile range (IQR; 25th and 75th percentiles) and compared by the Wilcoxon signed-rank test. The relationship between post-thaw seminal ORP and sperm parameters was examined using Spearman's correlation coefficient test. Statistical significance was considered at $p$ value $<.05$.

\section{3 | RESULTS}

Out of 35 fresh semen samples that were initially examined, 10 samples were excluded as they were not suitable for ORP testing ( 3 samples with azoospermia, 3 samples with severe oligozoospermia, and 4 samples with leukocytospermia). The remaining 25 samples were included in the analysis. The median (IQR) of post-thaw sperm concentration $\left[25(20,45) \times 10^{6} / \mathrm{ml}\right]$ and TSC $\left[12.5(10.0,17.5) \times 10^{6}\right]$ is significantly reduced as compared to the pre-freeze values [50 $(40,90) \times 10^{6} / \mathrm{ml}, p<.001 \& 120(90,250) \times 10^{6}, p<.001$ respectively]. The post-thaw values of seminal leucocyte counts [0.1 (0.0, $0.2)]$ are significantly lower than the pre-freeze values $[0.2(0.1,0.3)$; $p=.002]$.

Table 1 presents comparisons of sperm parameters (TM\%, PM\% and the percentage of normal sperm forms) and seminal ORP levels between the pre-freeze and post-thaw samples. The median (IQR) values for the CSR were $21.7 \%$ (11.2\%, 31.9\%). A highly significant positive correlation is observed between the pre-freeze and the post-thaw seminal ORP levels $(r=.67 ; p<.001)$.

A significant negative correlation is found between the prefreeze seminal ORP levels and the pre-freeze values of TSC $(r=-.59$; $p=.002)$. However, the pre-freeze seminal ORP levels are neither correlated with the pre-freeze TM\% $(r=-.08 ; p=.69)$ nor with prefreeze PM\% $(r=-.01 ; p=.95)$. Similarly, the pre-freeze seminal ORP 
levels are neither correlated with the post-thaw TM\% $(r=-.13$; $p=.59)$ nor with post-thaw PM\% $(r=-.08 ; p=.67)$.

On the other hand, the post-thaw seminal ORP levels are negatively correlated with the post-thaw TM\% ( $r=-.50 ; p=.02)$, PM\% $(r=-.41 ; p=.03)$, TSC $(r=-.60 ; p=.03)$ and CSR $(r=-.52 ; p=.01)$.

\section{4 | DISCUSSION}

Reactive oxygen species play an important role in sperm damage during cryopreservation (Chatterjee \& Gagnon, 2001). Variations in semen quality during different stages of cryopreservation are related to an imbalance between seminal antioxidant and oxidant status (Lone et al., 2018). The current study shows significantly higher post-thaw seminal ORP levels as compared to pre-freeze values, thus indicating OS in the post-thaw samples. Such observation agreed with the conclusion of a previous study that cryopreservation induces OS in cryopreserved semen samples (Thomson et al., 2009).

The median value of pre-freeze seminal ORP, in this study, does not exceed the cut-off value for normal semen $\left(\leq 1.34 \mathrm{mV} / 10^{6}\right.$ sperm $/ \mathrm{ml}$ ) that was recently established by Agarwal, Roychoudhury, et al. (2016) and Agarwal, Sharma, et al. (2016). The finding of normal pre-freeze values of seminal ORP, in the current study, may be attributed to (a) the exclusion of semen samples with high potential of oxidant production (i.e. leucocytospermia) and (b) the lack of semen samples with poor sperm parameters. The median values for the prefreeze sperm parameters and seminal leucocytic counts, in the current study, are consistent with the reference values of normal semen that were set by the WHO in 2010 (WHO, 2010). These findings may explain the lack of a significant correlation between the pre-freeze seminal ORP values and the pre-freeze sperm motility indices (TM\% and $\mathrm{PM} \%$ ).

The cryopreservation process, in this study, resulted in an approximately threefold increase in the levels of seminal ORP in the post-thaw samples. Such observation is important particularly in the light of the significant lowering of the post-thaw sperm TM\% and PM\% as compared to the pre-freeze values. The significant reduction of TM\% and PM\% in the post-thaw samples may also explain the lowering of CSR. Such observation is in line with the findings of previous reports (Lee, Lee, Wu, Hsu, \& Hsu, 2012; Oberoi, Kumar, \& Talwar, 2014).

The current study also indicates significant negative correlations between the post-thaw seminal ORP levels and the values of post-thaw TM\%, PM\%, TSC and CSR. Therefore, it can be speculated that OS mediates the detrimental effects of the freezing procedure for post-thaw sperm quality. In a previous study, ROSproducing subjects had significantly lower post-thaw sperm motility and vitality compared to ROS-free subjects (Mazzilli et al., 1995).

The freezing process has been shown to reduce the antioxidant capacity of spermatozoa, thus increasing their vulnerability to oxidative damage (Di Santo, Tarozzi, Nadalini, \& Borini, 2012). In the latter study, the authors have indicated that poor sperm motility in postthaw samples is due to oxidative damage to the plasma membrane and axonemal structures. Spermatozoa are particularly susceptible to the damage caused by excess ROS due to low antioxidants in their cytoplasm (Agarwal, Saleh, \& Bedaiwy, 2003. Therefore, efficient antioxidant systems are required to protect the sperm against oxidative damage (Aitken \& Fisher, 1994).

A recent study showed a higher potential for lipid peroxidation in cryopreserved spermatozoa as compared to fresh spermatozoa (Lone et al., 2018). Another study has suggested that the detrimental effects of cryopreservation on sperm function are due to increased production of ROS (Medeiros et al., 2002). In the latter study, the authors referred to the relationship between OS of cryopreserved spermatozoa and decreased fertility potential.

Despite interesting findings of the current study, few limitations are noted: (a) the exclusion of 10 semen samples that were not suitable for ORP testing resulted in a relatively small sample size. However, the included sample $(n=25)$ is sufficient for the purpose of this study; (b) the fertility status of the study population was undetermined (i.e. the study included men attending the infertility clinic for initial fertility evaluation); (c) the cross-sectional nature of the study precluded us from the follow-up of the participants to correlate the results to their reproductive outcomes. Studies that compare seminal ORP levels and sperm quality in cryopreserved semen samples from infertile men versus men with proven fertility may help address those issues; and (d) the influence of increased post-thaw seminal ORP on other indices of sperm quality such as sperm nuclear DNA was not tested in this study.

\section{5 | CONCLUSION}

In conclusion, sperm cryopreservation is associated with a significant elevation of seminal ORP and reduction of sperm TM\%, PM\% and CSR. Future research should focus upon the control of excess ROS generation and/or enhancement of antioxidant protection during the freeze-thaw process. Such efforts may help reduce the potential sperm cryo-damage and improve sperm quality following cryopreservation.

\section{ACKNOWLEDGEMENTS}

Authors would like to thank Dr. Gulfam Ahmad, PhD, Sydney, Australia, and Dr. Banafsheh Nikmehr, PhD, Tehran University of Medical Sciences, for their help with the revision of this manuscript.

\section{CONFLICT OF INTEREST}

None.

\section{ORCID}

Ramadan Saleh (iD https://orcid.org/0000-0003-0503-3533

Ralf Henkel iD https://orcid.org/0000-0003-1128-2982

Ashok Agarwal iD https://orcid.org/0000-0003-0585-1026 


\section{REFERENCES}

Agarwal, A., Roychoudhury, S., Bjugstad, K. B., \& Cho, C. L. (2016). Oxidation-reduction potential of semen: What is its role in the treatment of male infertility? Therapeutic Advances in Urology, 8, 302-318.

Agarwal, A., Saleh, R., \& Bedaiwy, M. A. (2003). The role of reactive oxygen species in the patho-physiology of human reproduction. Fertility and Sterility, 79, 829-843. (Review).

Agarwal, A., Sharma, R., Roychoudhury, S., Du Plessis, S., \& Sabanegh, E. (2016). MiOXSYS: A novel method of measuring oxidation reduction potential in semen and seminal plasma. Fertility and Sterility, 106, 566-573.

Aitken, R. J., Buckingham, D. W., \& Harkiss, D. (1993). Use of a xanthine oxidase free radical generating system to investigate the cytotoxic effects of reactive oxygen species on human spermatozoa. Journal of Reproduction and Fertility, 97, 441-450.

Aitken, R. J., \& Fisher, H. (1994). Reactive oxygen species generation and human spermatozoa: The balance of benefit and risk. Bio-assays, 16, 259-267.

Alvarez, J. G., \& Storey, B. T. (1995). Differential incorporation of fatty acids into and peroxidative loss of fatty acids from phospholipids of human spermatozoa. Molecular Reproduction and Development, 42, 334-346.

Amidi, F., Pazhohan, A., Shabani, N. M., Khodarahmian, M., \& Nekoonam, S. (2016). The role of antioxidants in sperm freezing: A review. Cell and Tissue Banking, 17, 745-756.

Anger, J. T., Gilbert, B. R., \& Goldstein, M. (2003). Cryopreservation of sperm: Indications, methods and results. Journal of Urology, 170, 1079-1084.

Bucak, M. N., Tuncer, P. B., Sarı̈zzkan, S., Başpınar, N., Taşpınar, M., Coyan, K., ... Oztuna, D. (2010). Effects of antioxidants on postthawed bovine sperm and oxidative stress parameters: Antioxidants protect DNA integrity against cryodamage. Cryobiology, 61, 248-253.

Chatterjee, S., \& Gagnon, C. (2001). Production of reactive oxygen species by spermatozoa undergoing cooling, freezing and thawing. Molecular Reproduction and Development, 59, 451-458.

Di Santo, M., Tarozzi, N., Nadalini, M., \& Borini, A. (2012). Human sperm cryopreservation: Update on techniques, effect on DNA integrity, and implications for ART. Advances in Urology, 2012, 1-12. https:// doi.org/10.1155/2012/854837

Kumar, A., Prasad, J. K., Mustapha, A. R., Amin, B. Y., Din, O., Katiyar, R., ... Ghosh, S. K. (2018). Reduction of dissolved oxygen in semen extender with nitrogen gassing reduces oxidative stress and improves post-thaw semen quality of bulls. Animal Reproduction Science, 197, 162-169.
Lee, C. Y., Lee, C. T., Wu, C. H., Hsu, C. S., \& Hsu, M. I. (2012). Kruger strict morphology and post-thaw progressive motility in cryopreserved human spermatozoa. Andrologia, 44, 81-86.

Lone, S. A., Prasad, J. K., Ghosh, S. K., Das, G. K., Balamurugan, B., \& Verma, M. R. (2018). Study on correlation of sperm quality parameters with antioxidant and oxidant status of buffalo bull semen during various stages of cryopreservation. Andrologia. https://doi. org/10.1111/and.12970

Mazzilli, F., Rossi, T., Sabatini, L., Pulcinelli, F. M., Rapone, S., Dondero, F., \& Gazzaniga, P. P. (1995). Human sperm cryopreservation and reactive oxygen species (ROS) production. Acta Europaea Fertilitatis, 26, 145-148.

Medeiros, C. M., Forell, F., Oliveira, A. T., \& Rodrigues, J. L. (2002). Current status of sperm cryopreservation: Why isn't it better? Theriogenology, 57, 327-344.

Oberoi, B., Kumar, S., \& Talwar, P. (2014). Study of human sperm motility post cryopreservation. Medical Journal, Armed Forces India, 70, 349-353.

Sharma, R. K., \& Agarwal, A. (1996). Role of reactive oxygen species in male infertility. Urology, 48, 835-850. (Review).

Sharma, R., Kattoor, A. J., Ghulmiyyah, J., \& Agarwal, A. (2015). Effect of sperm storage and selection techniques on sperm parameters. Systems Biology in Reproductive Medicine, 61, 1-12.

Sieme, H., Oldenhof, H., \& Wolkers, W. F. (2016). Mode of action of cryoprotectants for sperm preservation. Animal Reproduction Science, 169, 2-5.

Thomson, L. K., Fleming, S. D., Aitken, R. J., De luliis, G. N., Zieschang, J. A., \& Clark, A. M. (2009). Cryopreservation-induced human sperm DNA damage is predominantly mediated by oxidative stress rather than apoptosis. Human Reproduction, 24, 2061-2070.

Watson, P. F. (2000). The causes of reduced fertility with cryopreserved semen. Animal Reproduction Science, 60-61, 481-492.

World Health Organization (2010). WHO laboratory manual for the examination and processing of human semen (5th ed.). Geneva, Switzerland: WHO Press.

How to cite this article: Saleh R, Elsuity M, Henkel R, Agarwal

A. High levels of oxidation-reduction potential in frozenthawed human semen are significantly correlated with poor post-thaw sperm quality. Andrologia. 2020;52:e13608. https:// doi.org/10.1111/and.13608 